\title{
Maximizing Broadcast and Multicast Traffic Load through Link-Rate Diversity in Wireless Mesh Networks
}

\author{
Chun Tung Chou ${ }^{1}$, Bao Hua Liu ${ }^{2}$, Archan Misra ${ }^{3}$ \\ ${ }^{1}$ School of Computer Science and Engineering, University of New South Wales, Sydney, NSW 2052, Australia \\ 2 Thales Australia - Joint Systems, Garden Island, NSW 2011, Australia \\ ${ }^{3}$ IBM T. J. Watson Research Center, Hawthorne, New York, USA \\ Email: ctchou@cse.unsw.edu.au, Michael.Liu@thalesgroup.com.au, archan@us.ibm.com
}

\begin{abstract}
This paper studies some of the fundamental challenges and opportunities associated with the network-layer broadcast and multicast in a multihop multirate wireless mesh network (WMN). In particular, we focus on exploiting the ability of nodes to perform link-layer broadcasts at different rates (with correspondingly different coverage areas). We first show how, in the broadcast wireless medium, the available capacity at a mesh node for a multicast transmission is not just a function of the aggregate pre-existing traffic load of other interfering nodes, but intricately coupled to the actual (sender, receiver) set and the link-layer rate of each individual transmission. We then present and study four alternative heuristic strategies for computing a broadcast tree that not only factors in a flow's traffic rate but also exploits the wireless broadcast advantage (WBA). Finally, we demonstrate how our insights can be extended to multicast routing in a WMN, and present results that show how a treeformation algorithm that combines contention awareness with transmission rate diversity can significantly increase the total amount of admissible multicast traffic load in a WMN.
\end{abstract}

\section{INTRODUCTION}

Wireless Mesh Networks (WMNs) are regarded as a potentially disruptive telecommunications access technology for a variety of applications [5]. While recent deployment experiences (e.g., the Roofnet [8] and TFA [9] projects) attest to WMN's promise of low deployment cost and greater fault tolerance, the traffic load transportable over a multi-hop wireless path continues to be a significant bottleneck. It is becoming increasingly clear that a WMN should utilize the multi-rate capability at the link/MAC layers, especially as off-the-shelf 802.11-based cards dynamically adjust the link transmission rate to SNR variations. However, most enhancements to WMN operation, such as contention-aware channel assignment [22] or channel diversity-aware routing metrics [14], have focused on capacity improvement for unicast flows.

As part of our ongoing work on the Aiolos project [1], we focus explicitly on the case of broadcast and multicast traffic, and consider how WMN features impact the throughput of such point-to-multipoint flows. We believe that high-speed

This work was supported by the Australian Research Council Discovery Project grant DP0664791

This work was done when Dr. Bao Hua Liu was employed as a Senior Research Associate at the University of New South Wales, Australia.
WMNs will enable many exciting broadcast/multicast consumer applications (such as IP-TV or local content delivery, streaming of rich sensor feeds from security/traffic cameras, and multi-player games), and that it is thus important to analyze their performance in such mesh environments. Our previous work has focused purely on a a single broadcast flow and includes [13], where we used link-layer rate diversity to reduce the broadcast latency (defined as the maximum delay between the transmission of a packet by the source node and its eventual reception by all receivers) in a single-channel WMN, and [19], where we exploited the greater spatial concurrency available in multi-radio, multi-channel WMNs.

In this paper, we consider the implications of having multiple broadcast or multicast flows present in a single-channel WMN and address the following two questions:

- How does the potential transmission rate diversity impact the notion of how much broadcast traffic load can be feasibly accommodated on a specific data path?

- What sort of routing heuristics can increase the amount of broadcast/multicast traffic loads that a WMN can accommodate, and how much benefit does the link-rate diversity offer over the conventional approach of performing linklayer broadcasts at the base rate?

In particular, we focus on algorithms that consider the multirate operation of individual WMN, the traffic load of a new multicast flow, and the existing load from prior existing flows to construct the corresponding broadcast/multicast tree. We assume that each transmitting node can alter its linklayer broadcast transmission rate, which implicitly alters the broadcast's transmission range (or set of 'covered' receivers). While the current $802.11 \mathrm{a} / \mathrm{b} / \mathrm{g}$ standards mandate the broadcast transmission of control frames (e.g. RTS/CTS/ACK) at the lowest possible rate (e.g., $1 \mathrm{Mbps}$ for $802.11 \mathrm{~b}$ and $6 \mathrm{Mbps}$ for 802.11a), transmission rates for broadcast data are currently implementation-specific.

Not surprisingly, the creation of an individual broadcast/multicast tree depends directly on the interplay between the choice of a node's transmission rate, the resulting packet routing topology, and the consequent level of channel contention among neighboring nodes. For a broadcast flow where the sender generates $t$ bits/sec of traffic, an intermediate node 
$X$ in a feasible broadcast/multicast tree must be able to access the shared channel for a sufficient time to transmit at least $t$ bits/sec. A rate choice of $R$ bits/sec not only implies that node $X$ must access the channel for the fraction $\frac{t}{R}$, but also implicitly alters the degree of contention. For example, a faster rate $R$ may reduce the 'airtime held' by node $X$, but also reduces the coverage area of the broadcast, implying the need for additional transmissions (thus raising the level of contention) by a larger subset of downstream neighbors. Besides incorporating the impact of such link-layer rate diversity, a distribution tree for a newly arriving multicast flow should "route around" existing hot-spots (pockets of high contention from existing transmissions), so as to maximize the total amount of multicast traffic supported on the WMN. This problem is unique and different from prior work on load-aware multicasting in wired networks (e.g., [12]), since the algorithms also need to exploit the 'wireless broadcast advantage' (WBA) (to minimize the number of independent transmissions), as well as reduce the broadcast latency.

\section{A. Key Contributions of This Paper}

Our key objective is that each broadcast or multicast flow in a WMN should only use the minimally feasible network resources, without violating the feasibility constraints at any node on the data path, so as to maximize the total amount of broadcast or multicast traffic load that the network may simultaneously transport. Given this objective, this paper makes the following three contributions:

1) It shows how the feasibility of a particular link-layer broadcast is a function of not just the existing and incoming traffic load, but also of the chosen link-layer transmission rate.

2) For network-wide broadcast traffic, it presents and evaluates four heuristic tree construction algorithms that exploit transmission rate diversity, WBA and the residual capacity of the network (after taking into account the existing traffic load) to increase the amount of total traffic load that a WMN can carry.

3) For the practically important case of multicast traffic, it presents and evaluates a heuristic algorithm for tree construction that exploits transmission rate diversity, WBA and the residual capacity of the network. The proposed algorithm admits $30-40 \%$ more traffic than algorithms that use the base rate for all its link-layer transmissions.

The rest of this paper is organized as follows. Section II reviews the relevant related work. Section III details the unique interference-related capacity constraints for link layer transmissions. Section IV describes the heuristic algorithms and performance results for broadcast traffic, both via analytical techniques (using Matlab) and discrete event simulations (using Qualnet [2]). Similarly, Section V describes and evaluates the heuristic algorithm for resource-aware multicast in WMNs. Finally, Section VI concludes the paper with the important observations and discussion of open work.

\section{RELATED WORK}

A siginificant body of research in MANETs (Mobile Ad Hoc Networks) has researched efficient network layer multicast and broadcast, typically focusing on metrics such as energy consumption [10][24], the number of transmissions (which is equivalent to energy consumption if transmission power cannot be adjusted) [21] or the overhead in route discovery and management [16]. For WMN scenarios, where the mesh nodes are largely static (e.g., rooftop or electric pole mounted) and often powered from AC outlets, the total acceptable traffic load is a more critical performance metric than routing overhead or energy. QoS-aware MANET multicast routing algorithms have so far focussed on improving the delivery reliability (by either using resource reservation over multiple wireless paths (e.g., [6]), or constructing a delivery mesh instead of a tree (e.g., [23])), rather than focusing on the opportunities and challenges associated with link rate diversity and interference.

The problem of high throughput routing in WMN has been studied only for the case of unicast flows. The authors of [14] proposed a routing metric which can be used for a multichannel, multi-hop WMN. The proposed WCETT metric takes different transmission rates into account by having WCETT inversely proportional to the transmission rate. The work in [7] shows that if the interference range is infinity, then the unicast routing path that minimizes the total path delay will also maximizes the throughput between the source and destination. To deal with multi-rate links, [7] defines the rate-dependent medium-time metric (MTM), which measures the time it takes to transmit a packet over a multi-rate links including the transmission delay, overheads of the RTS/CTS/ACK frames and channel contention. In contrast to our focus on the network layer, the problem of maximizing the MAC-layer throughput for multicast transmissions (in the presence of different quality links and stability constraints) has been analyzed in [11].

Our earlier work in [13] has studied the problem of low broadcast latency in multirate WMNs. In particular, we presented an algorithm, based on the concept of weighted connected dominating set (WCDS), that explicitly balances the wireless broadcast advantage (WBA) with rate diversity to achieve low-latency network-wide broadcast. However, [13] focused only on a single broadcast flow and does not address the problem of how individual flows should be routed to maximize the total admissible volume of broadcast/multicast traffic in the presence of inter-flow and intra-flow interference.

\section{INTERFERENCE MODELLING AND FEASIBILITY ANALYSIS FOR RATE-DIVERSE TRANSMISSIONS}

In this section, we present the impact of interference on the feasibility of broadcast flows for a single-channel WMN. The analysis presented here explains how a candidate node on the routing tree for a new broadcast flow $F_{j}$ (with an associated offered load of $L_{j}$ ) can determine if it may feasibly forward the traffic for this flow using a link-layer broadcast rate $\rho$. This feasibility analysis will thus directly affect the formation of the broadcast forwarding tree (to be presented in Section IV).

We represent WMN as a graph $G(V, E)$, with the mesh nodes forming the vertices and the edge representing the link 


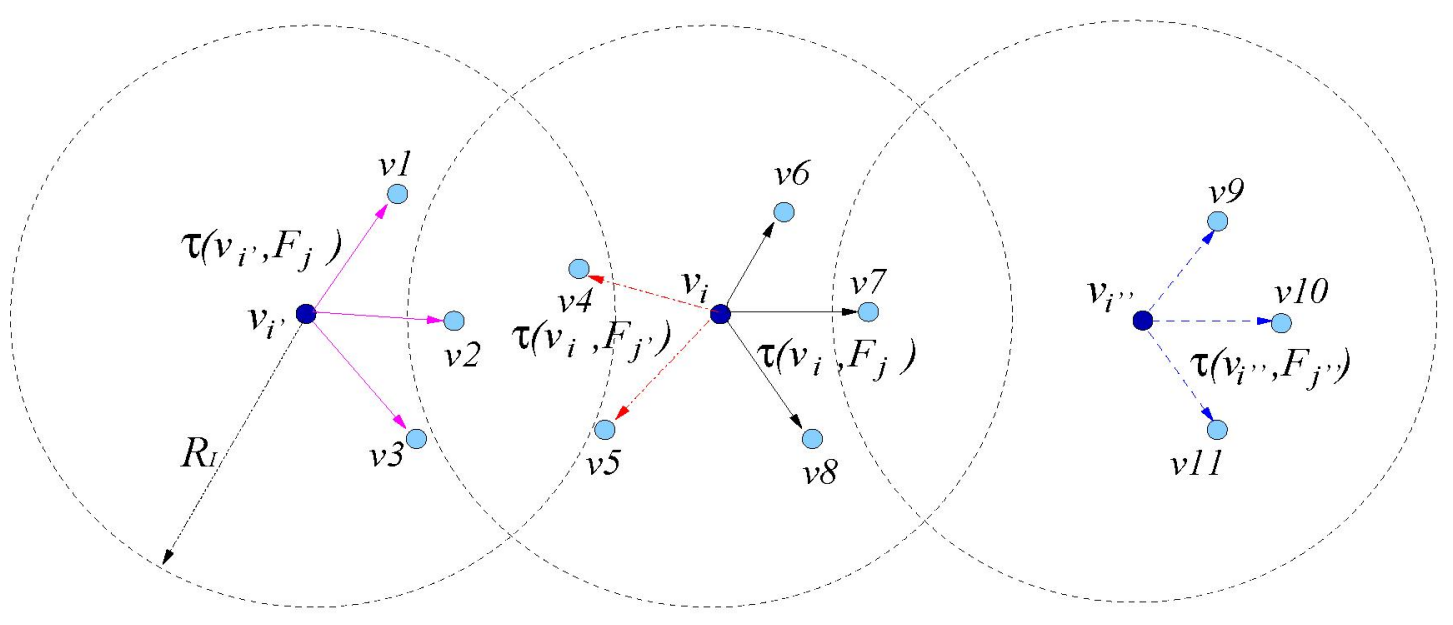

Fig. 1. Example showing the interference among multiple transmissions.

between two neighboring nodes. Each node can transmit at one of the $k$ available rates $R=\left\{\rho_{1}, \rho_{2}, \ldots, \rho_{k}\right\}$ where $\rho_{1}>\ldots>\rho_{k}$. Let $d\left(\rho_{i}\right)$ denotes the transmission range for rate $\rho_{i}$. We assume that all nodes use the same transmission power for all transmission rates. In addition, we assume that a binary disk packet reception model, the trade-off between link-layer transmission rate and transmission range means $d\left(\rho_{i}\right)<d\left(\rho_{j}\right)$ if $\rho_{i}>\rho_{j}$. A link $\left(v_{a}, v_{b}\right) \in E$ exists only if the distance $d\left(v_{a}, v_{b}\right)$ between nodes $v_{a}$ and $v_{b}$ is less than $d\left(\rho_{1}\right)$, and is associated with a rate $\rho_{v_{a}, v_{b}}$, the fastest feasible rate on $\left(v_{a}, v_{b}\right)$. We denote multiple incoming pointto-multipoint flows as $F_{1}, F_{2}, F_{3}, \ldots, F_{j}, \ldots$, each with traffic load $L_{1}, L_{2}, L_{3}, \ldots, L_{j}, \ldots$ (where the traffic is modelled as a fluid arrival process ${ }^{1}$ ). We assume a binary interference model, where a transmitting node $v_{a}$ is said to interfere with the reception of a node $v_{b}$ if and only if $d\left(v_{a}, v_{b}\right)<\kappa \times d\left(\rho_{1}\right)$, where $\kappa>1$. The distance $\kappa \times d\left(\rho_{1}\right)$ is known as the interference range.

A point-to-multipoint broadcast or multicast flow in a WMN can be realised by a forwarding tree whose non-leaf node will re-transmit the packets. We will therefore model a point-tomultipoint flow as a collection of a number of atomic linklayer multicast transmissions carried out by the non-leaf nodes of the forwarding tree. Each of these link-layer multicast transmissions is characterised by a transmission rate and its link-layer multicast recipients.

Definition 1: A link-layer multicast transmission $\tau\left(v_{i}, F_{j}\right)$ on node $v_{i}$ for flow $F_{j}$ is a two-tuple:

$$
\tau\left(v_{i}, F_{j}\right) \triangleq\left\{\rho\left(v_{i}, F_{j}\right), N\left(v_{i}, F_{j}\right)\right\}
$$

where $\rho\left(v_{i}, F_{j}\right) \in R$ denotes the transmission rate used by node $v_{i}$ for $F_{j}$, and $N\left(v_{i}, F_{j}\right)$ denotes the set of currently uncovered downstream neighbors that node $v_{i}$ would cover, if it transmitted at rate $\rho\left(v_{i}, F_{j}\right)$ (i.e., the set of nodes $\left\{v_{l}\right.$ : $d\left(v_{i}, v_{l}\right) \leq d\left(\rho\left(v_{i}, F_{j}\right)\right)$ and $v_{l}$ is currently uncovered $\left.\}.\right)$

\footnotetext{
${ }^{1}$ Our analysis, which is aimed at understanding the fundamental issues associated with multi-rate transmissions, assumes that $L_{i}$ represents the total traffic load of $F_{i}$, such that $\frac{L_{i}}{R}$ represents the total transmission time. For precise computation, $L_{i}$ should be adjusted to include the various overheads (network, MAC, PHY) associated with a specific transmission technology.
}

Note that by exploiting the WBA, the node $v_{i}$ can reach all the nodes in the set $N\left(v_{i}, F_{j}\right)$ in one transmission at rate $\rho\left(v_{i}, F_{j}\right)$.

\section{A. Definition and Properties of Broadcast Interference}

Given the broadcast nature of the wireless medium, the transmission $\tau\left(v_{i}, F_{j}\right)$ will interfere (or, equivalently, cannot occur simultaneously) with a set of other transmissions. In general, this set of interfering transmissions include transmissions by node $v_{i}$ itself (i.e., transmissions for other flows where $v_{i}$ is a non-leaf node), as well as transmissions by nearby interfering nodes. The inter-node interference for transmission $\tau\left(v_{i}, F_{j}\right)$ with a transmission by another node $\tau\left(v_{\hat{i}}, F_{\hat{j}}\right)$ occurs when $\tau\left(v_{i}, F_{j}\right)$ interferes with the reception by any of the recipients $N\left(v_{\hat{i}}, F_{\hat{j}}\right)$, or $\tau\left(v_{\hat{i}}, F_{\hat{j}}\right)$ interferes with the reception by any of the recipients $N\left(v_{i}, F_{j}\right)$. In particular, note that $F_{\hat{j}}$ may equal $F_{j}$ - i.e., there may be intra-flow interference caused by different nodes on the tree for $F_{j}$.

Definition 2: For any transmission $\tau\left(v_{i}, F_{j}\right)$, the interference set Inter $\left(\tau\left(v_{i}, F_{j}\right)\right)$ denotes the set of other transmissions that cannot occur in parallel with $\tau\left(v_{i}, F_{j}\right)$.

Figure 1 illustrates the nature of interference among several link-layer multicast transmissions, where $R_{I}=\kappa \times d\left(\rho_{1}\right)$ represents the interference range. There are three transmitting nodes $v_{i}, v_{i^{\prime}}, v_{i^{\prime \prime}}$ and four multicast transmissions $\tau\left(v_{i}, F_{j}\right)$, $\tau\left(v_{i}, F_{j^{\prime}}\right), \tau\left(v_{i^{\prime}}, F_{j}\right), \tau\left(v_{i^{\prime \prime}}, F_{j^{\prime \prime}}\right)$. The uncovered downstream neighbors of these four link-layer multicast transmissions are as follows $N\left(v_{i}, F_{j}\right)=\left\{v_{6}, v_{7}, v_{8}\right\}, N\left(v_{i}, F_{j^{\prime}}\right)=\left\{v_{4}, v_{5}\right\}$, $N\left(v_{i^{\prime}}, F_{j}\right)=\left\{v_{1}, v_{2}, v_{3}\right\}, N\left(v_{i^{\prime \prime}}, F_{j^{\prime \prime}}\right)=\left\{v_{9}, v_{10}, v_{11}\right\}$. We can see that $\tau\left(v_{i}, F_{j}\right)$ interferes with $\tau\left(v_{i^{\prime}}, F_{j}\right)$ at node $v_{2}$. $\tau\left(v_{i}, F_{j^{\prime}}\right)$ and $\tau\left(v_{i}, F_{j}\right)$ interferes with each other since they have the same transmitting node $v_{i} . \tau\left(v_{i^{\prime \prime}}, F_{j^{\prime \prime}}\right)$ interferes with $\tau\left(v_{i}, F_{j}\right)$ at node $v_{7}$. These interference constraints imply that the multicast transmission $\tau\left(v_{i}, F_{j}\right)$ cannot happen simultaneously with any of the other three multicast transmissions. Also note that the interference effects are not symmetric - e.g., while $\tau\left(v_{i^{\prime \prime}}, F_{j^{\prime \prime}}\right)$ interferes with $\tau\left(v_{i}, F_{j}\right)$ (at node $\left.v_{7}\right), \tau\left(v_{i}, F_{j}\right)$ does not cause any interference to any of the receivers of $\tau\left(v_{i^{\prime \prime}}, F_{j^{\prime \prime}}\right)$. 


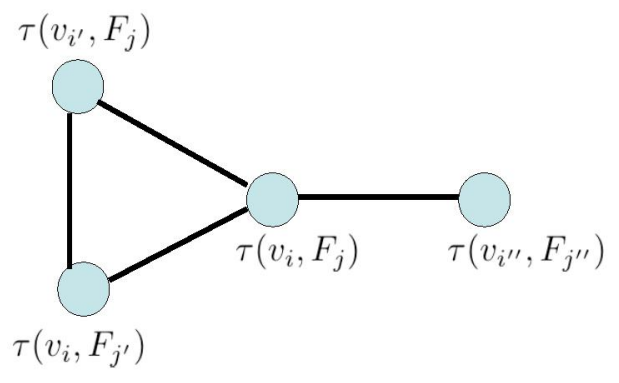

Fig. 2. Conflict graph of the multicast transmissions depicted in Figure 1.

The interference relationships among the transmissions in Figure 1 can be represented by a conflict graph (CG) shown in Figure 2. The nodes of the CG are the link-layer multicast transmissions and an edge exists between two nodes in the CG if their associated transmissions interfere with each other. CGs have also been used in modelling interference of unicast transmissions in [18]. However, the number of nodes in a multicast CG is far larger than that of a unicast CG. Let $\Delta_{i}$ denote the out-degree of node $v_{i}$, then the number of nodes in a unicast $\mathrm{CG}$ and multicast $\mathrm{CG}$ are, respectively, $\sum_{v_{i} \in V} \Delta_{i}$ (which is equal to the number of directed edges in the graph) and $\sum_{v_{i} \in V}\left(2^{\Delta_{i}}-1\right)$.

Given a number of transmissions, it can readily be proved that these transmissions can take place simultaneously if and only if they belong to an independent set ${ }^{2}$ of the CG. It was proved in [18] that a set of transmissions is feasible (or schedulable) if and only if it lies in the polytope of the independent sets of the CG. However, it is generally not feasible to apply this result in practice since the complexity to compute all independent sets grows exponentially with the number of nodes (which is already very large for a multicast CG).

In order to be able to determine a feasibility of a set of multicast transmissions, we will instead use a sufficient but not necessary condition. This condition may appear to be restrictive but our discrete event simulation shows that it can accurately predict the number of admission flows. We begin with a definition.

Definition 3: The transmission time fraction (TTF) of a link-layer multicast transmission $\tau\left(v_{i}, F_{j}\right)=$ $\left\{\rho\left(v_{i}, F_{j}\right), N\left(v_{i}, F_{j}\right)\right\}$ is:

$$
\operatorname{TTF}\left(\tau\left(v_{i}, F_{j}\right)\right)=\frac{L_{j}}{\rho\left(v_{i}, F_{j}\right)}
$$

We are now ready to state our admissibility condition.

Theorem 1: For a wireless mesh network with $p$ point-tomultipoint flows $F_{1}, \ldots, F_{p}$. Flow $F_{j}$ has a load of $L_{j}$ and

\footnotetext{
${ }^{2}$ Given a graph $(V, E)$ where $V$ is the set of nodes and $E$ is the set of edges. An independent set $I$ is a subset of $V$ such that no two elements in $I$ are connected by an edge.
}

whose forwarding tree is $T_{j}$. Let $\mathcal{N} \mathcal{L}\left(T_{j}\right)$ denote the set of non-leaf nodes for tree $T_{j}$. A sufficient condition for the flows $F_{1}, \ldots, F_{p}$ to be feasible is

$$
\begin{gathered}
\operatorname{TTF}\left(\tau\left(v_{i}, F_{j}\right)\right)+ \\
\sum_{\tau\left(v_{\hat{i}}, F_{\hat{j}}\right) \in \operatorname{Inter}\left(\tau\left(v_{i}, F_{j}\right)\right)} \operatorname{TTF}\left(\tau\left(v_{\hat{i}}, F_{\hat{j}}\right)\right) \leq 1
\end{gathered}
$$

for all $v_{i} \in \mathrm{NL}\left(T_{j}\right)$ and for all $F_{j}$.

Proof: This theorem is a generalisation of Theorem 1 in [17] to the case of multi-rate multicast transmissions.

The proof is similar to that in [17] and is omitted.

An important consequence of our analysis is that determination of the true feasibility of a particular flow requires the maintenance of flow-specific state for each of the separate transmissions. In the next section, we shall see how this complicates the formation of a broadcast tree, by requiring each node to essentially maintain awareness of each distinct transmissions within its interference set. Subsequently, in Section $\mathrm{V}$, we shall develop a less accurate node-centric feasibility metric for case of multicast flows.

\section{HEURISTIC BROADCAST ALGORITHMS}

We first present the generic principle for the formation of a broadcast tree for a newly incoming flow. We assume there are $j-1(j \geq 1)$ broadcast trees $\left\{T_{1}, \ldots, T_{j-1}\right\}$ already defined for the $\left\{F_{1}, \ldots, F_{j-1}\right\}$ flows in the network and describe the process of constructing the tree $T_{j}$ for flow $F_{j}$. The broadcast tree formulation is top-down - i.e., we start from the source and selectively add new nodes to the broadcast tree.

The objective of the algorithms is to create efficient delivery trees in order to achieve the maximal feasible load that a WMN may admit. As the load for all the flows are $L_{1}, L_{2}, L_{3}, \ldots, L_{j}, \ldots$, the metric for evaluating the 'goodness' of an algorithm is given by:

$$
\sum_{j} L_{j} \quad j \in\{1,2,3, \ldots J\}
$$

where $F_{J}$ is the last flow to be feasibly admitted (satisfies the constraints of Equation 3 at all forwarding nodes) and $F_{J+1}$ cannot be feasibly admitted.

We defer for now the question of selection metric, i.e., the question of how to pick the next tree node given an existing set of nodes for the partial tree $T_{j}$. Rather, we first demonstrate the process of verifying whether a new node (transmitting at a specific rate to a set of child nodes) is feasible. Our philosophy is thus to incrementally build a top-down broadcast tree $T_{j}$ that is feasible at all times, avoiding the addition of any transmission $\tau\left(v_{i}, F_{j}\right)$ that violates Equations 3 .

Let us assume that a number of nodes have been selected as transmitting nodes for flow $F_{j}$ in previous tree construction steps. This means for a selected node $v_{i^{\prime}}$, the transmission rate $\rho\left(v_{i^{\prime}}, F_{j}\right)$ and the downstream neighbors $N\left(v_{i^{\prime}}, F_{j}\right)$ for transmission $\tau\left(v_{i^{\prime}}, F_{j}\right)$ have been determined. We are now trying to determine if node $v_{i}$ can be selected as next transmitting node, i.e., if $\tau\left(v_{i}, F_{j}\right)$ with a transmission rate $\rho\left(v_{i}, F_{j}\right)$ and downstream neighbor $N\left(v_{i}, F_{j}\right)$ can be permitted. To verify this process, we consider all the possible transmissions of $\tau\left(v_{i}, F_{j}\right)$ with transmission rates $\rho_{1}, \ldots, \rho_{k}$. For any $\rho_{l}, l=$ 
$\{1, \ldots, k\}$ to be feasible, it is essential that the corresponding airtime constraint for $\tau\left(v_{i}, F_{j}\right)$ be satisfied, i.e.,

$$
\frac{L_{j}}{\rho_{l}}+\sum_{\tau\left(v_{\hat{i}}, F_{\hat{j}}\right) \in \operatorname{Inter}\left(\tau\left(v_{i}, F_{j}\right)\right)} \operatorname{TTF}\left(\tau\left(v_{\hat{i}}, F_{\hat{j}}\right)\right) \leq 1
$$

Given our desire to try to 'pack' as many flows into the WMN, it is natural to prefer nodes where the residual airtime fraction is higher (nodes whose neighborhood is less busy). Accordingly, we define the metric residual transmission time fraction (RTTF) for rate $\rho_{l}$ associated with transmission $\tau\left(v_{i}, F_{j}\right)$ for flow $F_{j}$ at node $v_{i}$ as:

$$
\begin{array}{r}
\operatorname{RTTF}\left(\tau\left(v_{i}, F_{j}\right) \mid \rho_{l}\right)=1-\frac{L_{j}}{\rho_{l}}- \\
\sum_{\tau\left(v_{\hat{i}}, F_{\hat{j}}\right) \in \operatorname{Inter}\left(\tau\left(v_{i}, F_{j}\right)\right)} \operatorname{TTF}\left(\tau\left(v_{\hat{i}}, F_{\hat{j}}\right)\right)
\end{array}
$$

Note that, as before, the computation of $\operatorname{RTTF}\left(\tau\left(v_{i}, F_{j}\right) \mid \rho_{l}\right)$ is dependent on not just the choice of the node $v_{i}$, but also the associate rate $\rho_{l}$ (as $\operatorname{Inter}\left(\tau\left(v_{i}, F_{j}\right)\right)$ depends on $\left.\rho_{l}\right)$. It is also worth to note the difference between $\tau\left(v_{i}, F_{j}\right)$ in Equation 6 and $\tau\left(v_{i}, F_{j}\right)$ in Equation 3. $\tau\left(v_{i}, F_{j}\right)$ in Equation 3 is a fixed transmission and hence its rate and downstream neighbors have been determined. In contrast, $\tau\left(v_{i}, F_{j}\right)$ in Equation 6 is not fixed and we are in the process of determining if it is feasible on node $v_{i}$ with a possible rate $\rho_{l}$. For feasibility of the candidate transmission $\tau\left(v_{i}, F_{j}\right)$, we need to check that $\operatorname{RTTF}\left(\tau\left(v_{i}, F_{j}\right) \mid \rho_{l}\right) \geq 0$. Moreover, when selecting among alternative nodes for possible inclusion in the tree, we should clearly prefer "less congested nodes", i.e., nodes with higher $\operatorname{RTTF}\left(\tau\left(v_{i}, F_{j}\right) \mid \rho_{l}\right)$. Note that the $\operatorname{RTTF}\left(\tau\left(v_{i}, F_{j}\right) \mid \rho_{l}\right)$ computed in Equation (6) is a worst-case value. To see this, suppose that two transmissions $\tau\left(v_{i^{\prime}}, F_{j^{\prime}}\right)$ and $\tau\left(v_{i^{\prime \prime}}, F_{j^{\prime \prime}}\right)$ both interfere with $\tau\left(v_{i}, F_{j}\right)$, but not with each other and can thus happen concurrently. $\operatorname{RTTF}\left(\tau\left(v_{i}, F_{j}\right) \mid \rho_{l}\right)$ however assumes that none of its interfering transmissions can occur in parallel with one another.

\section{A. Heuristic Metrics for Broadcast Tree Formation}

Given our goal of maximizing the amount of admitted broadcast load, we should try to reduce the consumption of airtime by individual transmissions. In general, we thus want that (a) each transmission $\tau\left(v_{i}, F_{j}\right)$ by $v_{i}$ uses as high a transmission rate as possible, and (b) the number of transmissions be minimized. Clearly, these two desires are mutually conflicting, since a faster rate implies a smaller coverage area, and consequently a larger number of individual transmissions.

We now present four feasible metrics for computing the tree $T_{j}$ based on the notion of a connected dominating set (CDS). Recall that for a graph $G(V, E)$, a $\operatorname{CDS} Z$ of $G$ is a subset of $V$ such that (1) Every element (node) of $V \backslash Z$ is in the neighborhood of at least one node in $Z$; (2) The set $Z$ is connected. Among all the CDSs of $G$, constructing the one with the minimum cardinality (the minimum connected dominating set or MCDS) is known to be an NP-hard problem for a unit disk [15]. In this paper, we extend the WCDS (Weighted CDS) algorithm presented in [13] for constructing an MCDS-based broadcast tree in a multi-rate WMN. Note that WCDS itself was suggested purely for an individual flow, does not consider the effects of inter and intra-flow interference, and does not attempt to maximize the total amount of admitted broadcast traffic. Our heuristic algorithms start by making the source node $s$ for $F_{j}$ eligible to transmit, and setting $Z$ (denoting the set of covered nodes) to $\{s\}$. We say that a node is 'covered' if it is within the transmission range of a node $v \in Z$, given $v$ 's current link rate. In each round of the algorithm, we choose the $\tau\left(v_{i}, F_{j}\right)$ combination for a node $v_{i} \in Z$ that maximizes some objective function $f\left(\tau\left(v_{i}, F_{j}\right)\right.$ ) (and, of course, does not violate the constraints of Equations 3). Algorithm 1 illustrates the overall steps for all the algorithms, with the computation of $f\left(\tau\left(v_{i}, F_{j}\right)\right)$ being the sole point of difference among the four heuristics. In all cases, the tree formation process may terminate at an intermediate point if no additional feasible transmission is found. In such a case, we reject the admission of incoming flow $F_{j}$.

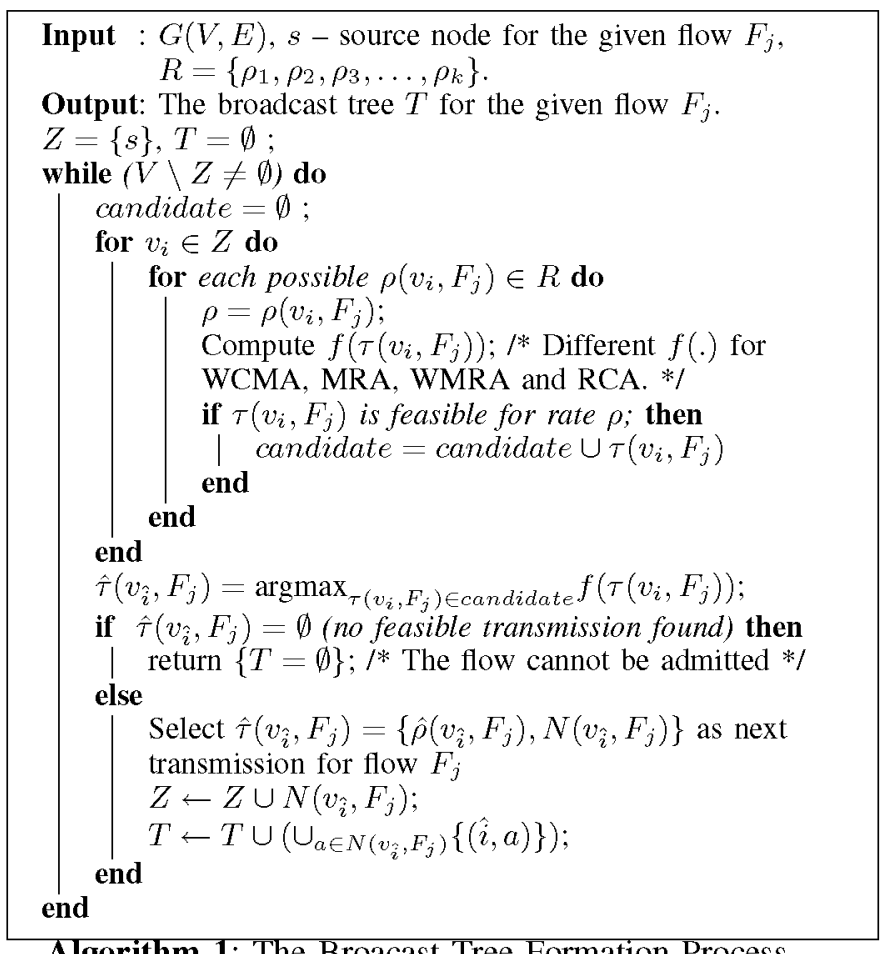

Algorithm 1: The Broacast Tree Formation Process

The first algorithm, called the Weighted Coverage Maximization Algorithm (WCMA), calculates the cost of a candidate transmission $\tau\left(v_{i}, F_{j}\right)$ as follows:

$$
f_{W C M A}\left(\tau\left(v_{i}, F_{j}\right)\right)=\left|N\left(v_{i}, F_{j}\right)\right| \times \rho\left(v_{i}, F_{j}\right)
$$

This is identical to the WCDS metric in [13], except for the additional step of verifying that the chosen rate satisfies the feasibility constraints.

The second algorithm considers only the effect of interference on a single transmission. The transmission rate is fixed with the lowest rate (e.g., $6 \mathrm{Mbps}$ for IEEE 802.11a radio). This algorithm is called the Maximum RTTF Algorithm (MRA) and tries to select the transmission that results in the maximum residual airtime. Accordingly, the cost of a 
candidate transmission $\tau\left(v_{i}, F_{j}\right)$ is given by:

$$
f_{M R A}\left(\tau\left(v_{i}, F_{j}\right)\right)=\operatorname{RTTF}\left(\tau\left(v_{i}, F_{j}\right) \mid \rho\left(v_{i}, F_{j}\right)\right)
$$

By selecting the (node, rate) combination with the largest $R T T F$ value, this heuristic tries to maximize the residual airtime, with the expectation that this will eventually allow more future transmissions to be accommodated.

The third algorithm, called the Weighted Maximum RTTF Algorithm (WMRA) balances the desire to select the transmission with the maximum residual airtime and higher transmission rate. The cost $f\left(v_{i}, F_{j}\right)$ is thus computed as:

$$
\begin{gathered}
f_{M R A}\left(\tau\left(v_{i}, F_{j}\right)\right)=\rho\left(v_{i}, F_{j}\right) \times \\
R T T F\left(\tau\left(v_{i}, F_{j}\right) \mid \rho\left(v_{i}, F_{j}\right)\right)
\end{gathered}
$$

Note that in WMRA, the rate selected must cover at least one uncovered neighbor.

Finally, the fourth algorithm, called the RTTF-Aware Coverage Algorithm $(R C A)$, computes the cost of $f\left(v_{i}, F_{j}\right)$ as follows:

$$
\begin{gathered}
f_{R C A}\left(\tau\left(v_{i}, F_{j}\right)\right)=\left|N\left(v_{i}, F_{j}\right)\right| \times \rho\left(v_{i}, F_{j}\right) \\
\times \operatorname{RTTF}\left(\tau\left(v_{i}, F_{j}\right) \mid \rho\left(v_{i}, F_{j}\right)\right)
\end{gathered}
$$

Intuitively, the RCA algorithm tries to balance the competing objectives of interference minimization (favoring nodes with larger $R T T F$ ), link rate maximization (to reduce broadcast latency), and coverage of currently uncovered nodes (favoring transmissions that cover more nodes in the broadcast tree).

\section{B. Idealized Performance Results for Broadcast Heuristics}

We first present the results of Matlab-based simulations (essentially assuming an ideal MAC layer) to understand the behavior of the various tree formation heuristics. We use the parameters given in Table I and $\kappa=1.7$ in our study. The transmission rates and minimum sensitivities shown in Table I are reproduced from IEEE 802.11a specifications [3]. The transmission range for each given rate is derived from Qualnet [2] with two-ray ground propagation model and fixed transmission power of $16 \mathrm{dBm} \mathrm{Bm}^{3}$. The results presented in this subsection correspond to means computed over 50 uniformly randomly generated network topologies. All network topologies are connected where the connectivity has been tested against the lowest transmission rate. Each network topology covers an area of $1 \mathrm{~km}^{2}$.

The study is conducted with all flows having identical load $L=0.1 \mathrm{Mbps}$. The network throughput is calculated as the product of the number of flows feasibly admitted and $L$. Figure 3 shows the comparative results of the four heuristic broadcast algorithms with $95 \%$ of confidence interval (All figures are plotted with $95 \%$ of confidence interval in the rest of the paper). Clearly, the throughput achieved by RCA outperforms the others. On average, RCA achieves 18.7\%, $78.1 \%$, and $7.8 \%$ of improvement than that of WCMA, MRA, and WMRA respectively. This demonstrates that the broadcast

${ }^{3}$ This is equivalent to $40 \mathrm{~mW}$, which is the standard maximum power for 5.15-5.25 GHz band [3].
TABLE I

RADIO RANGE FOR IEEE802.11A.

\begin{tabular}{|l|l|l|}
\hline $\begin{array}{l}\text { Transmission } \\
\text { Rate (Mbps) }\end{array}$ & $\begin{array}{l}\text { Minimum Sensi- } \\
\text { tivity }(\mathrm{dBm})\end{array}$ & $\begin{array}{l}\text { Transmission } \\
\text { Range }(\mathrm{m})\end{array}$ \\
\hline 6 & -82 & 170.62 \\
\hline 9 & -81 & 152.07 \\
\hline 12 & -79 & 120.79 \\
\hline 18 & -77 & 95.95 \\
\hline 24 & -74 & 67.93 \\
\hline 36 & -70 & 42.86 \\
\hline 48 & -66 & 27.04 \\
\hline 54 & -65 & 24.10 \\
\hline
\end{tabular}

capacity can be enhanced by choosing transmissions that balance the need for high link rates with greater per-transmission node coverage and low channel contention (as measured by the RTTF metric).

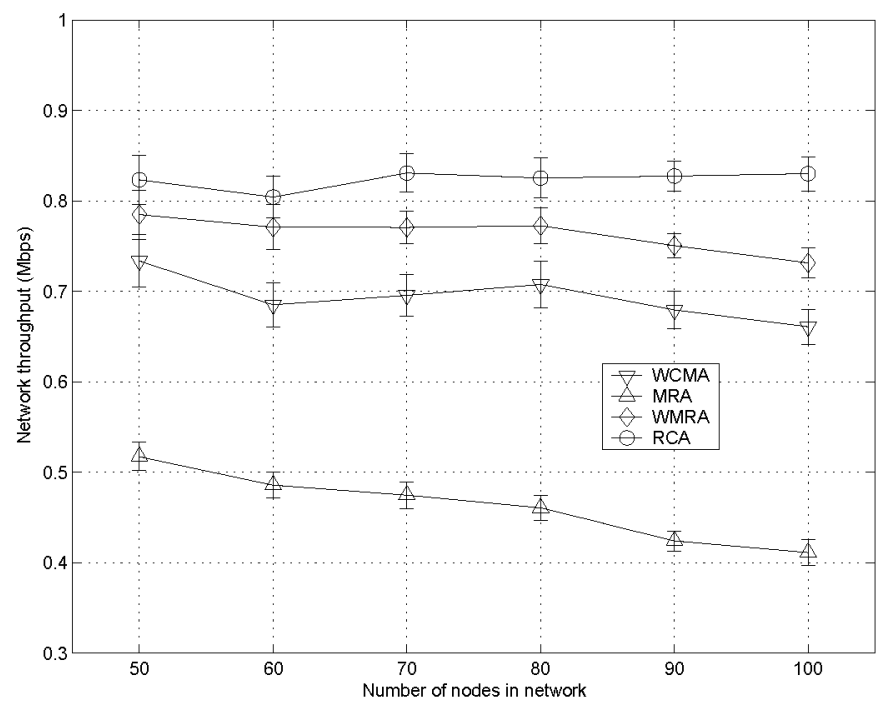

Fig. 3. Total admissible traffic with rate diversity.

\section{The effect of transmission rates on broadcast Capacity}

The aim of this section is to study how the network broadcast capacity depends on the choice of transmission rate. This question does not appear to have a trivial answer because of the trade off between transmission time and transmission coverage area. Although a higher rate transmission takes a shorter time, its coverage area is smaller. This means that it will take more higher rate transmissions to cover the same physical area and higher number of transmissions can also mean more contention to the channel.

In order to answer the above question, we used the ratediversity aware routing algorithms that we have proposed earlier but instead of using multiple transmission rates, each node is restricted to transmit at a single link-layer transmission rate. In our simulation, we used three broadcast algorithms: WCMA, MRA and RCA. For each algorithm, we carried out three sets of simulations where the nodes in each set of simulations used only one single rate. Three different rates were used: $6 \mathrm{Mbps}, 9 \mathrm{Mbps}$ and $12 \mathrm{Mbps}$. Note that network 
partitioning prevented us from using higher transmission rates. Note also that although reducing the network area can result in a connected network for higher transmission rates, the reduced network area becomes unsuitable for our study because the larger transmission range for lower transmission rate means most nodes are covered in a one-hop transmission.

Figure 4 shows the comparative performance of the three algorithms with different transmission rates. The results were obtained from the average of 50 random topologies with 150 nodes in each network. The figure shows that $9 \mathrm{Mbps}$ results in the highest network broadcast capacity. This shows that a higher link-layer transmission rate does not necessarily lead to a higher broadcast capacity. It was shown in $[4$, Theorem 8] that for the broadcast capacity $c$ of a multi-hop wireless network (in a $d$-dimensional cube) whose nodes use a singlelink layer transmission rate $\rho$ is bounded between:

$$
c_{1} \frac{\rho}{\max \left(1, \Delta^{d}\right)} \leq c \leq c_{2} \frac{\rho}{\max \left(1, \Delta^{d}\right)}
$$

where $c_{1}$ and $c_{2}$ are constants independent of the network parameters, and

$$
\Delta=\frac{d_{i}-d_{r}(\rho)}{d_{r}(\rho)}
$$

where $d_{i}$ is the interference range and $d_{r}(\rho)$ is the transmission range for transmission rate $\rho$. This shows that in a 2-dimensional cube, the broadcast capacity varies with transmission rate according to

$$
c \sim \frac{\rho}{\max \left(1,\left(\frac{d_{i}-d_{r}(\rho)}{d_{r}(\rho)}\right)^{2}\right)}
$$

By using the transmission rate and transmission range values given in Table $\mathbf{I}$ and $d_{i}=\kappa d\left(\rho_{1}\right)$ with $\kappa=1.7$, Figure 5 shows how broadcast capacity (13) varies with transmission rates. It shows that the broadcast capacity is highest when the $9 \mathrm{Mbps}$ transmission rate is being used. Our simulation results therefore agrees with the prediction derived from [4]. Our study therefore shows that a higher link-layer transmission rate does not necessarily translate to a higher broadcast capacity. If a single link-layer transmission rate is to be used to achieve high broadcast capacity, then equation (11) can be used to predict the best rate to be used. Note that although [4] presented formula (11), the question of how the tradeoff between transmission rate and transmission range (which rises from a constant transmission power assumption) affects the broadcast capacity was not discussed. In particular, note that our derivation assumes the same transmission power is used for all transmission rate.

The above discussion shows that some link-layer rates are better in realising a high broadcast capacity than the others when a single link-layer rate is to be used. What about in the multi-rate case? Will a certain subset of link-layer rates be better in realising high broadcast capacity? We study this question by performing simulations using three multirate broadcast algorithms WCMA, MRA and RCA. Instead of using all the transmission rate available in Table $\mathrm{I}$, we perform simulations where only rates from 6Mbps to $x$ Mbps are used where $x=6,9,12,18,24, \ldots$. The results are plotted

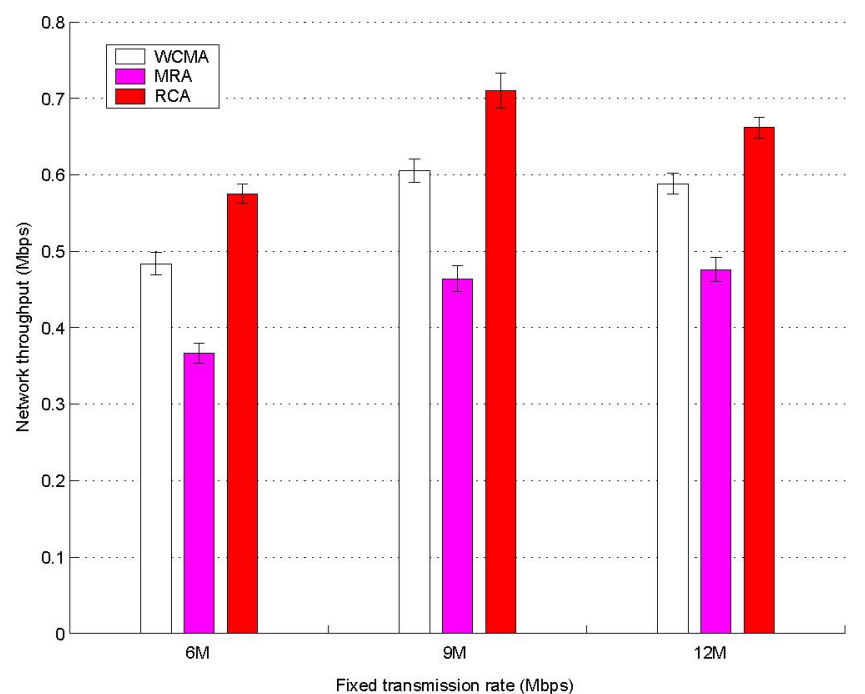

Fig. 4. Total admissible traffic with a single link-layer transmission rate.

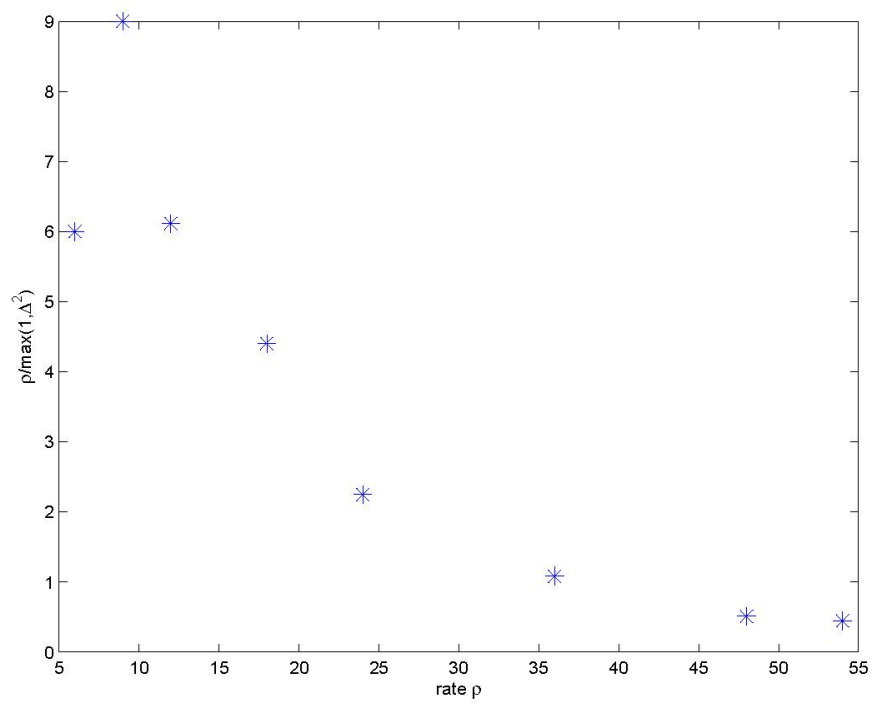

Fig. 5. The figure shows how broadcast capacity varies with transmission rate according to equation (13).

in Figure 6 where the $x$-axis indicates the highest transmission rate being used, e.g. $x$ equals to 24 means the rates $6,9,12,18$ and $24 \mathrm{Mbps}$ are available for the nodes to use. Figure 6 shows that the inclusion of higher transmission rates increases the broadcast capacity (which is understandable) but the inclusion of some higher rates does not seem to improve the broadcast capacity much. It is interesting to note in Figure 6 that the inclusion of $9 \mathrm{Mbps}$ gives the biggest increase in broadcast capacity. This suggests that equation (11) can be used as a guideline to determine which rates are to be included in the multi-rate case.

We note that the effect of link-layer rates on broadcast latency (i.e. the time it takes a source node to reach all nodes) in a multi-hop wireless mesh network was studied in [13], [20]. They show that if a single link-layer rate is to be 
used, then a higher rate may not result in lower broadcast latency. In fact, they show that the rate-area product (i.e. the product of transmission rate and transmission coverage area) is a good rule-of-thumb in determining how effective a transmission rate is in reducing broadcast latency in a 2 dimensional network. Note that the rate-area product is in fact the first order approximation of the right-hand-side of equation (13) for $\Delta>1$.

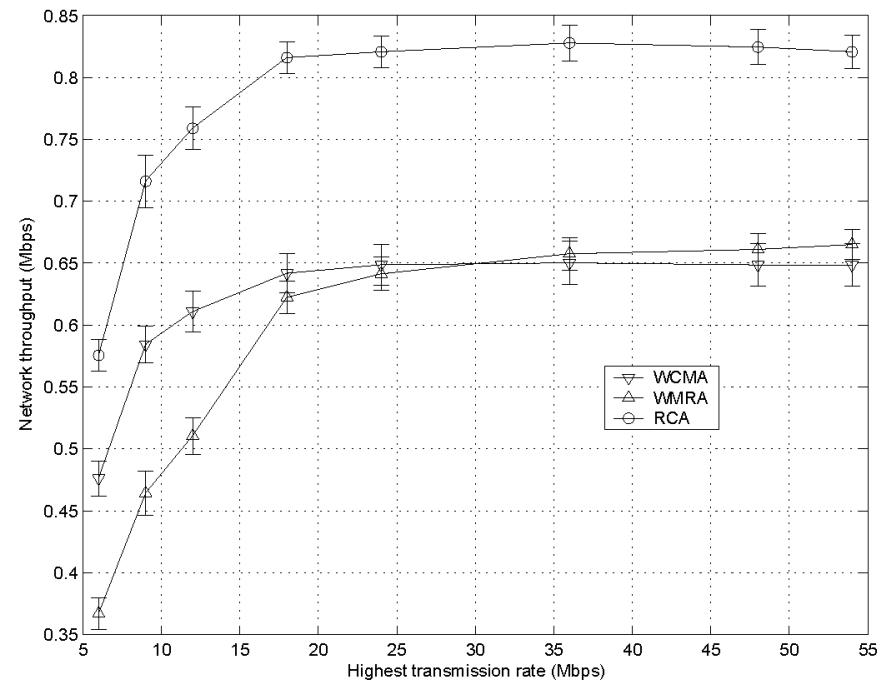

Fig. 6. Broadcast capacity.

\section{Simulations with IEEE 802.11 a}

Former Matlab-based studies assume idealized MAC where media contentions can be resolved perfectly. Moreover, the traffic load is modelled as a fluid arrival process. In a real WMN, messages are transmitted in packets. While network throughput is a critical measure for the performance of an algorithm, it makes no sense if the packet delivery ratio is too low. In order to study our proposed algorithms in a more realistic wireless environment, we fed the computed trees (with rate-diversity) into the discrete event simulator Qaulnet [2] and observe their performance ${ }^{4}$ with IEEE 802.11 a radio.

Figure 7-9 show three critical performance measures packet delivery ratio, network throughput, and broadcast latency. All plotted values are averaged over all flows on all nodes. Each simulation run is conducted for the period of 100 seconds. Figure 7 shows that the average packet delivery ratios for all algorithms are well above $95 \%$. It also shows that WMRA and RCA achieve very similar delivery ratio. WCMA achieves a slight higher performance compare to WMRA and RCA because it accommodates a slight smaller traffic load. MRA achieves the best performance since MRA accommodates much less traffic load compare to the others. We further observe that the average network throughput shown in Figure 8 closely mirrors Figure 3. This means that our computations of broadcast trees are indeed showing some

\footnotetext{
${ }^{4}$ Due to implementation issues, the generated packet load for each flow is slightly higher than $0.1 \mathrm{Mbps}$.
}

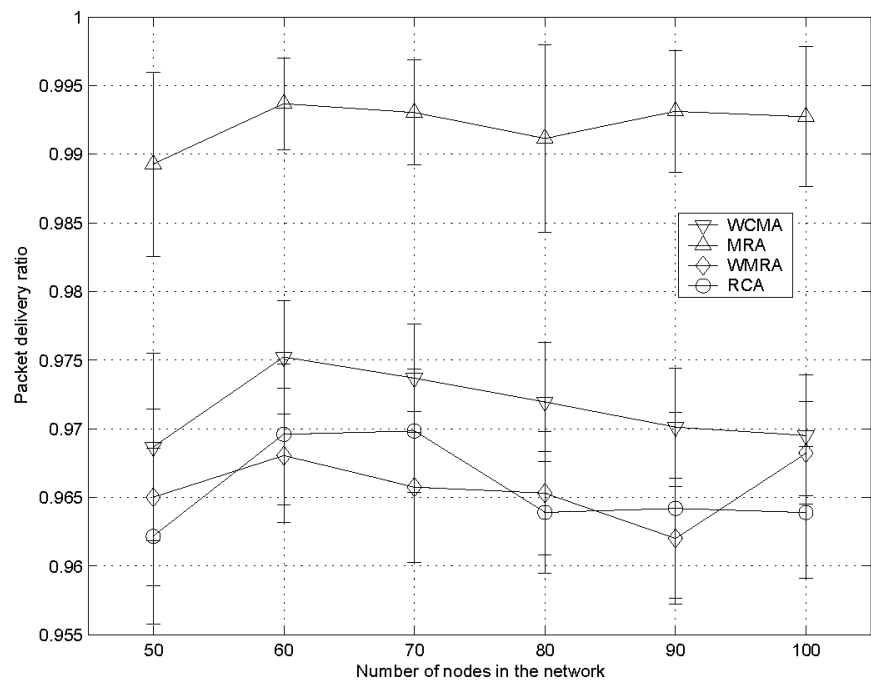

Fig. 7. Average packet delivery ratio via Qualnet.

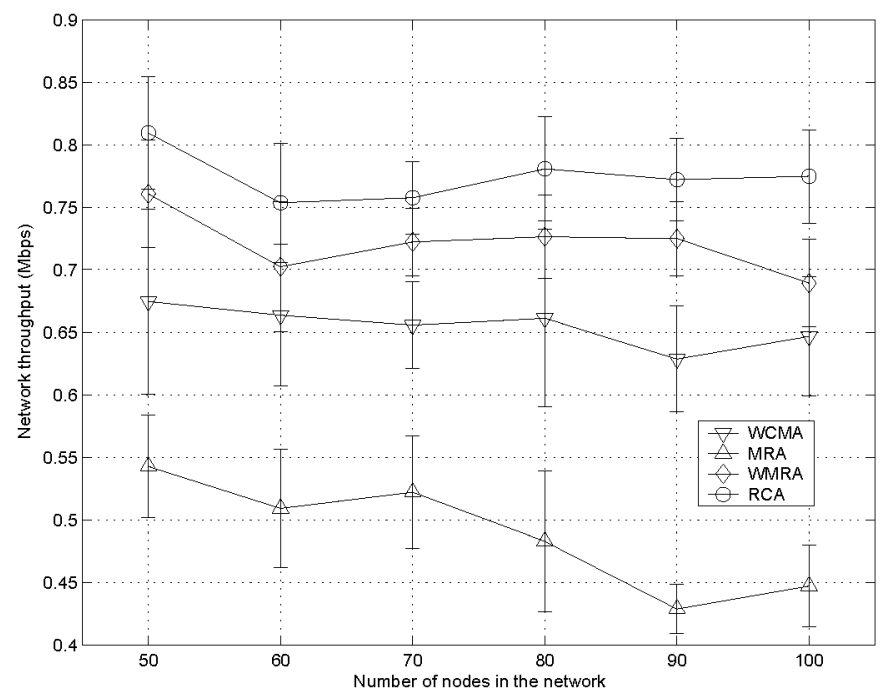

Fig. 8. Average network throughput via Qualnet.

"matching results" in 802.11 a environment. Figure 9 illustrates the average broadcast latency. It is understandable that WCMA and MRA achieves better performance compare to WMRA and RCA since they accommodate less traffic load, hence less media contention at MAC layer. An interesting observation of Figure 9 is that RCA outperforms WMRA although RCA in fact accommodates more traffic load than WMRA. The reason behind this behavior is that WMRA biased towards selecting faster transmission rate - but smaller transmission range which covers lesser number of nodes. This results a larger number of transmissions are required in order to achieve a network-wide broadcast, which leads to higher contention-induced delays and poor performance at MAC layer.

\section{RATE AND CONTENTION AWARE MULTICAST}

We now consider the more practical problem of building similar routing trees for multicast flows. Unlike all earlier work 


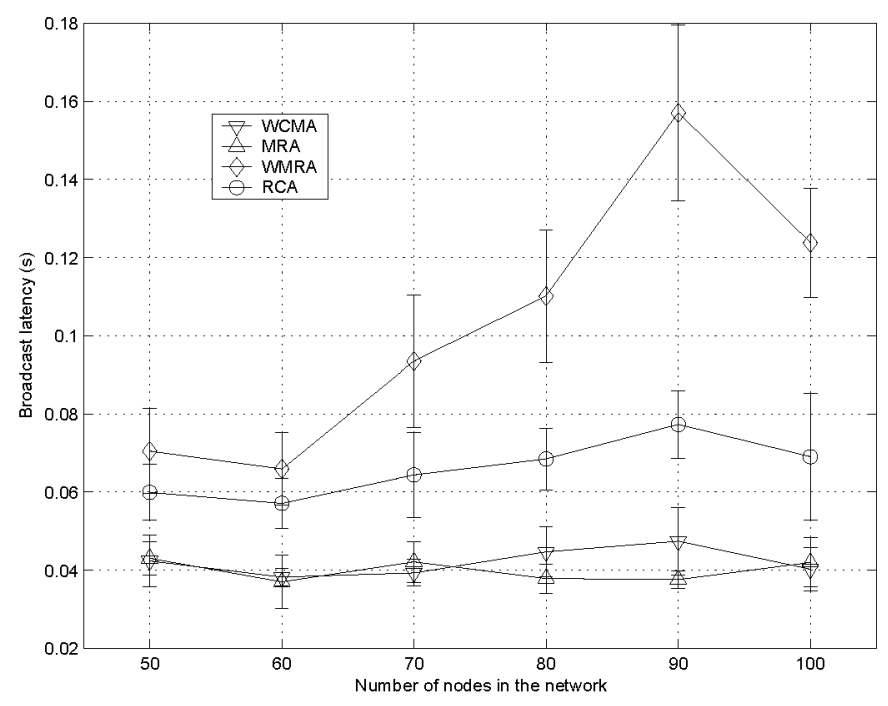

Fig. 9. Average broadcast latency via Qualnet.

on broadcasting, we aim to build a multicast tree that explicitly factors in three unique WMN features - (a) the ability of nodes to operate at different link rates; (b) the impact of interference on the available (bandwidth) capacity of a WMN node, and (c) the WBA. The key difficulty in extending the interferenceaware approach (embodied by the RTTF metric of Equation 6) to multicast flows is that the broadcast tree formation algorithms are greedy - i.e., they compute the tree starting at the source and greedily add nodes to the tree, corresponding to the "best subsequent" transmission. In contrast, the multicast tree cannot be built greedily, since nodes should only be added if they extend the tree towards one of the receivers. (Most distance-vector algorithms, such as Dijkstra, cannot solely compute the shortest path to a specific destination node $v_{d}$ from a source $v_{s}$, but instead, reconstruct the shortest path by backward traversal after computing a larger set of shortest paths). While one approach for multicasting may thus be based on pruning (i.e., first create the broadcast tree, and then simply prune all unnecessary edges), this is likely to be unsatisfactory. In particular, by assuming that all neighboring nodes needed to receive a transmission, the broadcast tree formation process may have incorrectly excluded some (link, rate) combinations.

Accordingly, we have devised the Rate and Contention Aware Multicast Algorithm (RCAM) (mathematically outlined in Algorithm 2) with the following intuition. The multicast tree will be constructed incrementally taking into account the rate, time fraction usage, and WBA. We assumed that the set of $Q$ multicast receivers $\left\{m r_{1}, \ldots, m r_{Q}\right\}$ are known at the start of the tree formation process. In the first step, we find the least-cost unicast path from source $s$ to any multicast recipient, say $m r_{\alpha}$, of the set of $Q$ receiver nodes, assuming a link cost $c\left(v_{a}, v_{b}\right)$ for any link $\left(v_{a}, v_{b}\right)$. In general, the higher the rate for the edge $\left(v_{a}, v_{b}\right)$, the smaller should be the link cost. However, to balance the link cost with the level of channel contention, $c\left(v_{a}, v_{b}\right)$ needs to also account for the amount of residual airtime in the neighborhood of $\left(v_{a}, v_{b}\right)$. The most accurate determination of this contention is given by the metric RTTF (see Equation 6), which however, depends on the precise receiver set for a specific transmission $\tau($.$) . As$ this is not possible for multicast as the relevant downstream receivers are not known a-priori, we instead define a flowindependent metric Cumulative Transmission Time Fraction $(C T T F)$ for a node $v_{i}$ as:

$$
\operatorname{CTTF}\left(v_{i}\right)=\sum_{l=1}^{j-1} \sum_{v_{m} \in V} \frac{L_{l}}{\rho\left(v_{m}, F_{l}\right)} I\left(v_{i}, v_{m}, F_{l}\right)
$$

where $I\left(v_{i}, v_{m}, F_{l}\right)$ is an indicator function that equals 1 (otherwise 0) if: ( $v_{m}$ is a transmitting node for tree $\left.T_{l}\right) \wedge$ ( $v_{m}$ or at least one of the receivers in $N\left(v_{m}, F_{l}\right)$ is within the interference range of $v_{i}$ ). In other words, CTTF $\left(v_{i}\right)$ defines the cumulative airtime usage (across all prior scheduled transmissions) in the interference range of $v_{i}$.

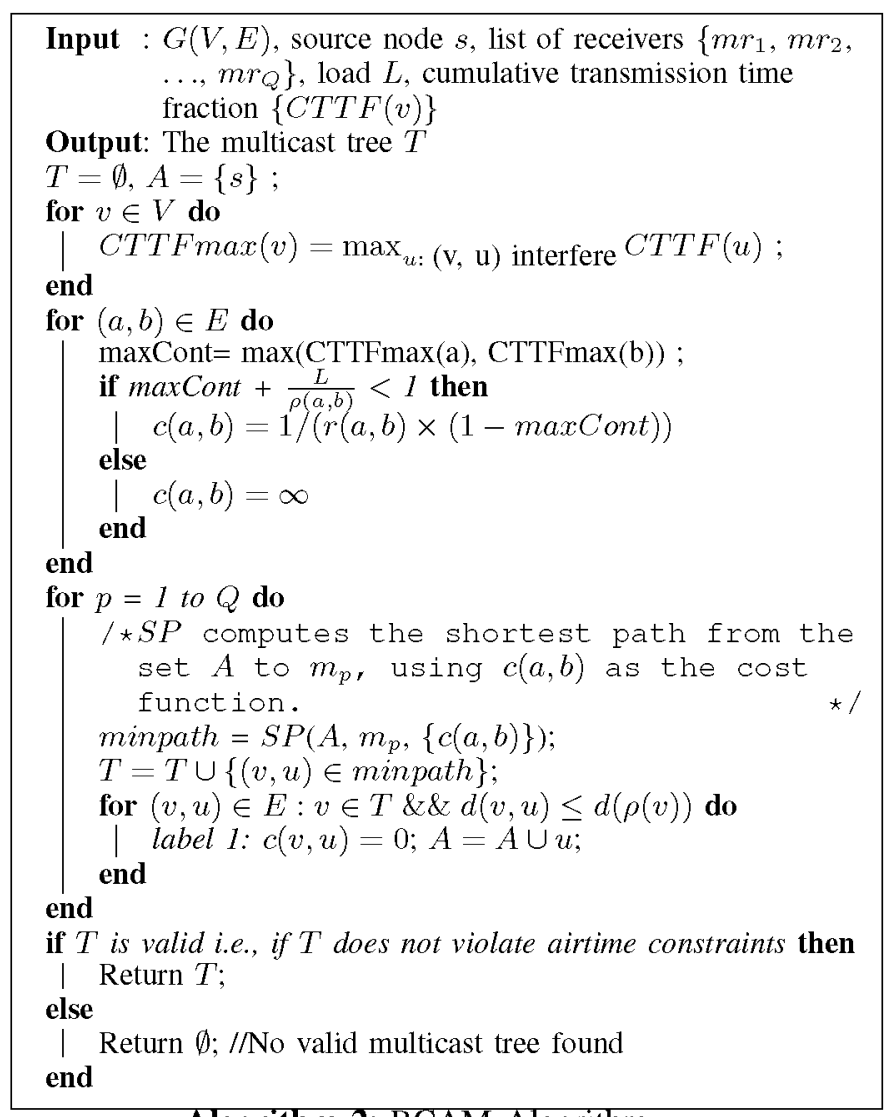

Algorithm 2: RCAM Algorithm.

To account for interference, the link cost $c\left(v_{a}, v_{b}\right)$ is modified to be a function of both the link speed $\rho\left(v_{a}, v_{b}\right)$ and the most critical airtime constraint in $v_{a}$ 's vicinity. Thus,

$$
\begin{array}{r}
c\left(v_{a}, v_{b}\right)=\frac{1}{\rho\left(v_{a}, v_{b}\right)} \times \\
\frac{1}{1-\max _{d\left(v_{a}, v_{l}\right) \leq \kappa \times d\left(\rho_{1}\right)} C T T F\left(v_{l}\right)}
\end{array}
$$

Moreover, if $\operatorname{CTTF}\left(v_{i}\right)+\frac{L_{j}}{\rho\left(v_{a}, v_{b}\right)}>1$, then $c(a, b)$ should equal $\infty$ to reflect the fact that this link, although physically present, is unusable due to airtime constraints.

While such a formulation accounts for the rate diversity, RCAM also needs to account for the WBA. In particular, if 
a node $v_{a}$ has already been chosen to as a forwarding node of the tree $T_{j}$, it follow that any node $v_{b}$ in the neighborhood of $\tau\left(v_{a}, F_{j}\right)=\left\{\rho\left(v_{a}, F_{j}\right), N\left(v_{a}, F_{j}\right)\right\}$, i.e., $v_{b} \in N\left(v_{a}, F_{j}\right)$, can receive the packet for free due to WBA. This is reflected by setting their cost $c\left(v_{a}, v_{b}\right)$ to 0 (label 1 in Algorithm 2) since this transmission is free due to WBA. (Note: The authors have also used this zero-cost setting technique to compute resilient multicast trees which exploit WBA in [25]). After this adjustment, the RCAM algorithm proceeds iteratively by selecting the next receiver node having the least-cost unicast path among the remaining receivers (e.g., selecting $m r_{\beta}$ next) and grafting this path onto the existing multicast tree (the set $A$ in Algorithm 2). To perform this grafting, RCAM selects the least-cost feasible path from the receiver to any member of $A$. Note that due to the inaccurate formulation of $\operatorname{CTTF}\left(v_{i}\right)$, it is possible that the final computed tree $T_{j}$ may actually be infeasible (i.e., it may violate one of the constraints of Equations 3. Accordingly, RCAM performs a final feasibility check on the whole tree $T$; if it is found to be infeasible, the entire multicast flow is rejected.

\section{A. Idealized Performance Results for Multicast Heuristics}

We first used Matlab-based studies (assuming an idealized MAC) to compare the multicast capacity achieved by RCAM in a WMN with two alternative algorithms that do not consider interference effects: (a) The Pruning algorithm, where the broadcast tree is first constructed (using Equation 7) and all unnecessary nodes are subsequently pruned. (b) The conventional shortest path tree (SPT) algorithm, where the tree is formed by merging the shortest unicast path (with a link's cost being the inverse of its transmission rate) from source to each individual multicast receiver.

Our primary metric of interest is the amount of multicast traffic that the algorithms can feasibly admit. To study the dependence of capacity on the number of multicast receivers per group $(Q), Q$ is chosen from $\{5,10,30\}$. For each flow, the source and receiver nodes are selected randomly from the set of WMN nodes. All flows have identical load $L=0.1 \mathrm{Mbps}$. Simulations are performed with 50 network topologies where each network topology has 400 nodes uniformly randomly distributed on a $1.5 \mathrm{~km} \times 1.5 \mathrm{~km}$ area. Figure 10 illustrates the simulation results.

From Figure 10, we see that, by considering both link rate diversity and interference-induced contention, RCAM outperforms WCMA and SPT. More importantly, the performance gains for RCAM are much greater when the number of multicast receivers are sparse $(59 \%$ improvement over SPT for $Q=5$ ) compared to dense receiver sets ( $23 \%$ improvement over WCMA for $Q=30$ ). Clearly, sparse multicast groups allow multicast trees to be routed around WMN 'hotspots' or bottlenecks, allowing more flows can be 'packed'. As $Q$ increases, it is more likely that receivers for a newly arriving flow will be located in an existing 'hotspot', leaving little choice to the routing protocol. Finally, we see that the total network throughput (measured purely as the sum of the sender load, and not weighted by the number of receivers per flow) decreases as $Q$ increases, since a larger value of $Q$ implies a greater overall use of airtime resources per flow.

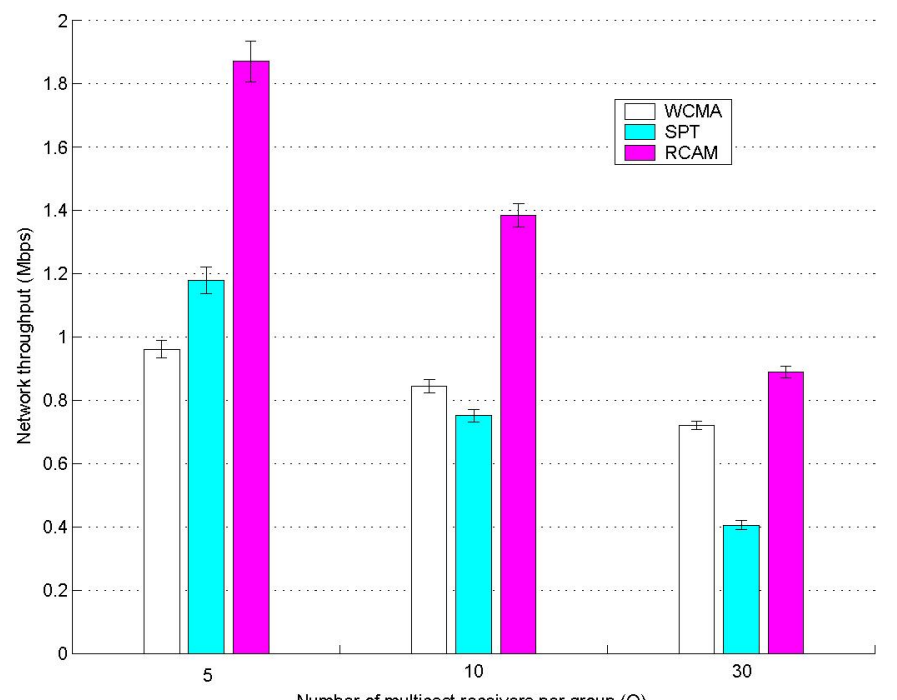

Fig. 10. Total admissible multicast traffic load.

\section{B. The effect of transmission rates on multicast capacity}

Similar to study carried out in Section IV-C on how a single link-layer transmission rate affects the broadcast capacity, in this section, we study how the choice of link-layer transmission rate impacts on multicast capacity. Figure 11 illustrates the comparative results of the three algorithms with different combinations of transmission rate and number of multicast receivers per group. Transmission rate that is higher than $12 \mathrm{Mbps}$ cannot be used since the network connectivity cannot be guaranteed. Figure 11 shows that algorithms with fixed transmission rate of $9 \mathrm{Mbps}$ achieve the highest performance, which is very close to the results as shown in Figure 10. But it is clear that our proposed heuristic RCAM algorithm outperforms the peak performance by exploiting the ratediversity. An interesting observation of Figure 11 is that the algorithms with fixed transmission rate of $12 \mathrm{Mbps}$ achieves worse performance than that of $9 \mathrm{Mbps}$. This means that the employment of higher rate may have negative impact on the network throughput.

\section{Simulations with IEEE 802.11 a}

Similar to the broadcast study, we conducted discrete event simulations via Qualnet for all the multicast algorithms and observed their performance. We measured similar parameters (e.g., packet delivery ratio, network throughput, multicast latency) but only the multicast receivers associated values have been used in the average calculation. Figure 12-14 illustrate the comparative results. We can see that the network throughput as shown in Figure 13 closely mirrors Figure 10, which means that our computation of multicast trees indeed showing "matching results" in 802.11a environment. Although RCAM achieves significant improvements in the network throughput compare to WCMA and SPT, we can see from Figure 12 and Figure 14 that the packet delivery ratio and multicast latency of RCAM did not deteriorate significantly. In fact, RCAM achieves similar performance compare to WCMA and 


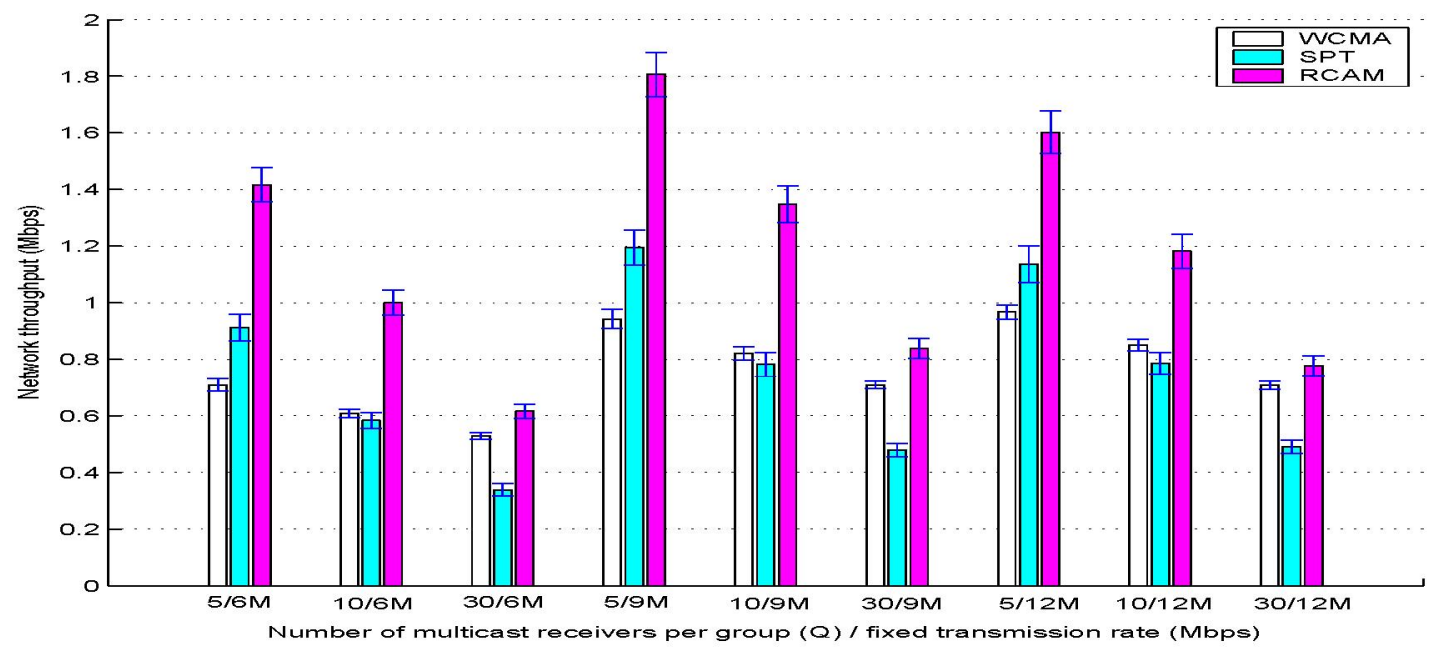

Fig. 11. Total admissible multicast traffic load with fixed transmission rates.

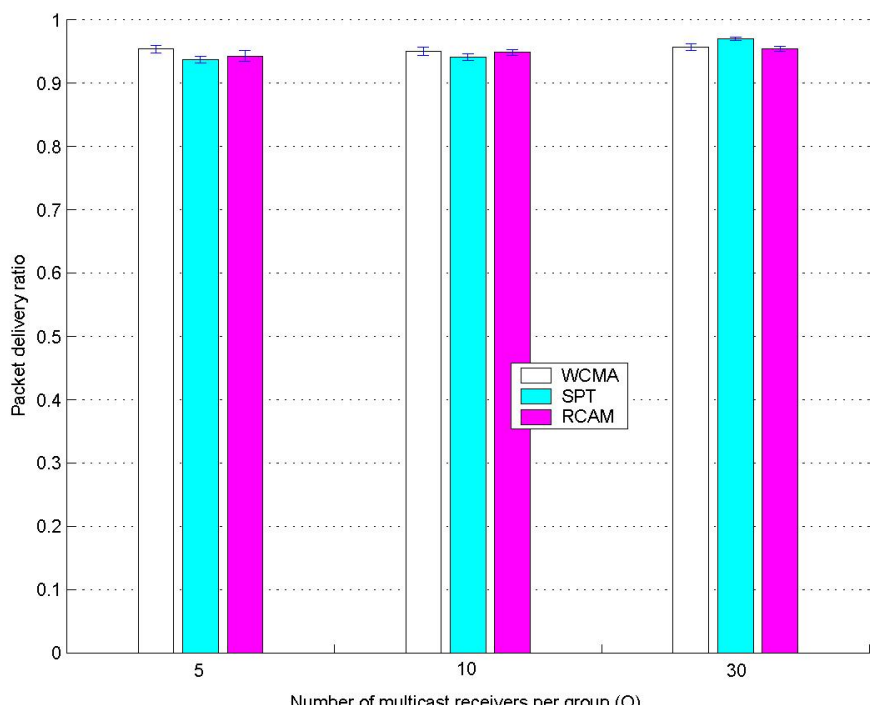

Fig. 12. Average packet delivery ratio via Qualnet.

SPT. In some cases, RCAM even achieves better performance. For example, RCAM has the lowest multicast latency with $Q=10$.

\section{CONCLUSIONS AND FUtURE WORK}

We have demonstrated that the combined consideration of link-rate diversity and channel interference can significantly increase the amount of broadcast/multicast traffic load that may be feasibly admitted and routed within a WMN. For network-wide broadcast traffic, the RCA heuristic algorithm provides up to $78 \%$ of improvement in the total broadcast capacity (total feasible load) by choosing transmissions that balance between high link rates, greater node coverage and low channel contention. For multicast flows, the RCAM algorithm is able to significantly enhance the amount of admissible multicast traffic on a WMN by exploiting both the transmission rate and the available (contention-free) airtime at individual

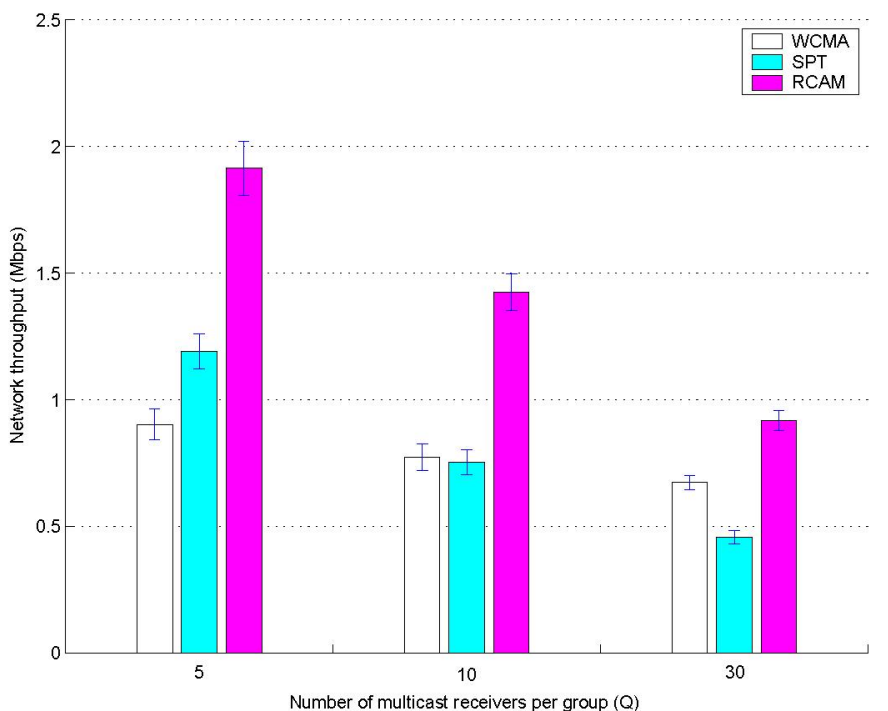

Fig. 13. Average network throughput via Qualnet.

nodes. In particular, results demonstrating large (e.g., $59 \%$ for $Q=5$ ) capacity gains for relatively sparse multicast groups are of great practical significance to many real-life applications (e.g., games, video-conferencing). In addition, our discrete events simulations with 802.11a radio via Qualnet shows decent "matching results" compare to the idealized studies.

While these are fundamental results, we are currently working to develop more practical, distributed tree formation algorithms based on the heuristics presented in this paper. The design of rate-diversity aware multicast tree formation algorithms for multi-radio, multi-channel wireless mesh nodes remains an open question for future research.

\section{REFERENCES}

[1] Efficient distribution of content in multi-rate, multichannel wireless mesh networks. The Aiolos Project, http://www.cse.unsw.edu.au/ aiolos/index.html. 


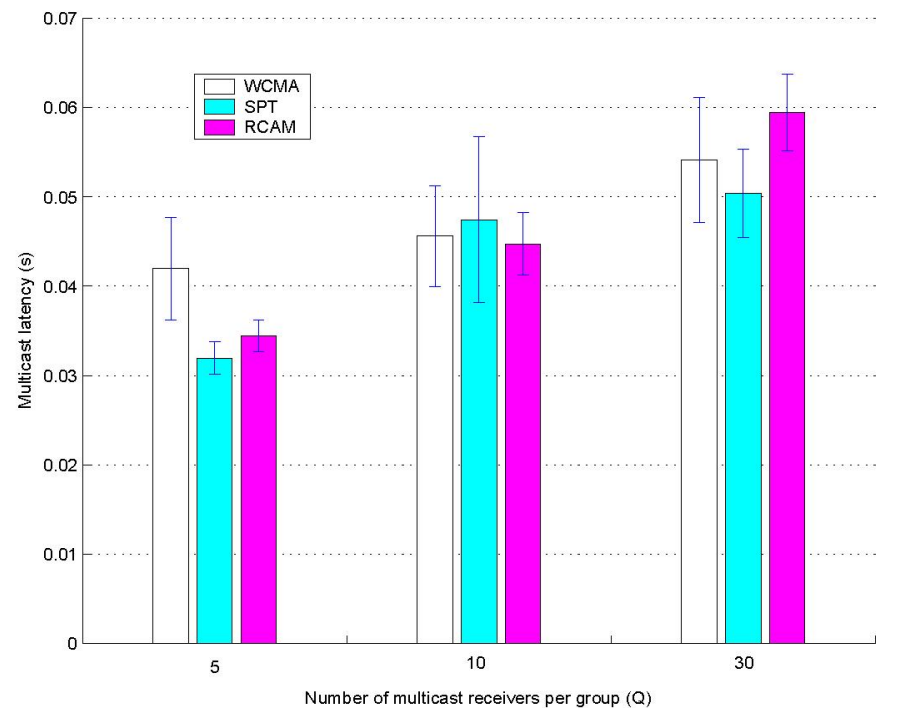

Fig. 14. Average multicast latency via Qualnet.

[2] Qualnet, http://www.qualnet.com.

[3] Wireless lan medium access control (mac) and physical layer (phy) specifications: High-speed physical layer in the $5 \mathrm{Ghz}$ band. IEEE Std 802.11a-1999', 1999.

[4] V. R. A. Keshavarz-Haddad and R. Riedi. Broadcast capacity in multihop wireless networks. 'Proc. ACM Mobicom, 2006.

[5] I. Akyildiz, X. Wang, and W. Wang. Wireless mesh networks: A survey, Elesevier Computer Networks, 47:445-487, 2005.

[6] T. Anker, D. Dolev, and Y. Eliaspur. Off-piste qos-aware routing protocol. Proc. of IEEE ICNP, pages 24-35, Oct. 2004

[7] B. Awerbuch, D. Holmer, and H. Rubens. High throughput route selection in multi-rate ad hoc wireless networks. Lecture Notes in Computer Science, 2004

[8] J. Bicket, D. Aguayo, S. Biswas, and R. Morris. Architecture and evaluation of an unplanned $802.11 \mathrm{~b}$ mesh network. Proc. of MOBICOM pages $31-42$, Aug. 2005

[9] J. Camp, J. Robinson, C. Steger, and E. Knightly. Measurementdriven deployment of a two-tier urban mesh access network. Proc. of ACM/Usenix Mobisys 2006., pages 96-109, 2006.

[10] J. Cartigny, D. Simplot, and I. Stojmenovic. Localized minimum-energy broadcasting in ad-hoc networks. Proc. of INFOCOM, 3:2210-2217, Mar. 2003

[11] P. Chaporkar and S. Sarkar. Wireless multicast: Theory and approaches. IEEE Trans. of Information Theory, 51(6):1954-1972, Jun. 2005.

[12] S. Chen, K. Nahrstedt, and Y. Shavitt. A qos-aware multicast routing protocol. IEEE Journal on Selected Areas in Communications (JSAC), 18(12):2580-2592, Dec. 2000

[13] C. T. Chou, A. Misra, and J Qadir Low latency broadcast in multirate wireless mesh networks. IEEE Journal on Selected Areas in Communications Special Issue on 'Multi-hop Wireless Mesh Networks', 24(11):2081-2091, Nov 2006.

[14] R. Draves, J. Padhye, and B. Zill. Routing in multi-radio, multi-hop wireless mesh networks. Proc. of Mobicom, pages 114-128, 2004.

[15] S. Guha and S. Khuller. Approximate algorithms for connected dominating set. Algorithmica, 20:347-387, 1998 .

[16] C. Gui and P. Mohapatra. Scalable multicasting in mobile ad hoc networks. Proc. of INFOCOM, 3:2119-2129, Mar. 2004

[17] R. Gupta, J. Musacchio and J. Walrand, Sufficient rate constraints for QoS Flows in Ad-Hoc Networks. Ad Hoc Networks, volume 5, issue 4 , pages 429-443, May 2007 ,

[18] K. Jain, J. Padhye, V.N. Padmanabhan and L. Qiu. Impact of interference on multi-hop wireless network performance. Proc of ACM Mobicom, pp. $66-80,2003$

[19] J. Qadir, A. Misra, and C. T. Chou. Minimum latency broadcasting in multi-radio multi-channel multi-rate wireless meshes. Proc. of IEEE Communications Society Conference on Sensor, Mesh and Ad Hoc Communications and Networks (SECON), Sep 2006.

[20] J. Qadir , C. T. Chou, and A. Misra. Exploiting rate diversity for broadcasting in wireless mesh networks. Proc. IEEE Conference on Local Computer Networks (LCN), Nov 2006.

[21] W. Lou and J. Wu. On reducing broadcast redundancy in ad hoc wireless networks. IEEE Trans. on Mobile Computing, 1(2):111-123. Apr. 2002

[22] J. Tang, G. Xue, and C. Chandler. Interference-aware routing and bandwidth allocation for QoS provisioning in multihop wireless networks. Wireless Communications and Mobile Computing Joumal, 5(8):933943,2005

[23] $\mathrm{H}$ Tebbe A. Kassler, and $\mathrm{P}$ Ruiz Qos-aware mesh construction to enhance multicast routing in mobile ad hoc networks. Proc. of InterSense, May 2006.

[24] J. E. Wieselthier, G. D. Nguyen, and A. Ephremides. On the construction of energy-efficient broadcast and multicast trees in wireless networks. Proc. of INFOCOM, 2:585-594, 2000.

[25] X. Zhao, C. T. Chou, J. Guo and S. Jha. Protecting Multicast Sessions inWireless Mesh Networks, 'Proc. IEEE Conference on Local Computer Networks (LCN). Nov 2006. 